\title{
A New Technique for Fast Detection of Progressive Faults
}

\author{
Fahmida Chowdhury and Bin Jiang
}

\author{
Dept. of ECE, P.O.Box 43890 \\ University of Louisiana at Lafayette \\ Lafayette, LA 70504-3890, USA \\ Email: \{fnchowdh;bin.jiang $\} @$ louisiana.edu
}

\begin{abstract}
In this paper, we propose a new technique for enhancing the residual signal in a way that reduces the rate of false alarms without introducing detection delay. This is achieved by eliminating most of the noise from the residual signal by performing an autoregressive modeling, where the model parameters are estimated in real time by an ordinary Kalman filter. Computer experiments using a Boeing 747 model are used to demonstrate the implementation of the proposed technique.
\end{abstract}

\section{INTRODUCTION}

Early detection and identification of faults (abnormal conditions) leads to increased reliability in complex engineering systems. Therefore, fault detection and identification (FDI) is an important and active research field. Published literature in the field contains many techniques for detecting faults: observers, parity space methods, eigenstructure assignment, parameter identification based approaches, etc. Useful results can be found in several excellent survey papers(Gertler, 1988; Frank et al., 2000) and books (e.g., Basseville and Nikiforov, 1993; Chen and Patton, 1999; Silvio et al., 2002). However, early detection of progressive (slowly developing and increasing) faults has really not received a lot of attention from the research community; only a few results on this issue have been reported until now (for example, see Demetriou and Polycarpou (1998), Parlos et al (2002)). Progressive faults are difficult to detect early, especially when measured (sensor) data are corrupted by noise. Application of the existing FDI techniques to progressive faults in the presence of measurement noise is complicated by the tradeoff between false alarms and missed detection. Typically, reducing the false alarm rate will result in detection delay. In this paper, we propose a technique that can reduce the rate of false alarm without sacrificing the speed of detection. For stochastic systems, FDI is typically based on statistical testing of the residuals. The basis of all statistical hypothesis testing is that in the normal (fault-free) case, the residuals constitute a random process with known statistical properties; when a fault occurs, the residuals constitute a different statistical process. The whole idea of fault detection is, then, exactly the same as detection of a change in statistical properties. During recent years, a lot of research has been done on FDI for stochastic systems (Chen and Patton, 1999; Keller, 1999; Chowdhury, 2000) - the common technique in all of these methods is the generation of a residual signal that can be used, with a high level of confidence, as an indicator of faults. Also, all FDI includes, in one form or another, tests of hypotheses that are applied to the fault indicator.

In all the hypothesis testing techniques, a trade-off is expected between the rate of false alarm and that of missed detection or detection delay. Too many false alarms make system operation rather unreliable, but trying to reduce the number of false alarms typically results in an increased probability of missed alarms, which translates into detection delays. Since false alarms are a direct result of the noisiness of the residuals, it is clear that techniques of reducing the noise level of the residuals, without losing the fundamental information regarding the presence of the fault, would be of great benefit.

In this paper, we propose a technique that extracts the useful signal from noisy residuals. This method would be especially useful for detecting slowly evolving faults in dynamic systems; the technique is based on an autoregressive (AR) modelling of the residuals. The residuals can be generated by any of the conventional methods; the only requirement is that during fault-free operation, they should be zero-mean and timewise uncorrelated random variables. We focus on progressive faults because in case of sudden faults the raw residuals are usually sufficient to detect the fault. Moreover, using the existing methods, when we try to reduce the number of false alarms by setting the detection threshold high (for example, at $3 \sigma$ : three times the standard deviation, the false alarm rate is $1 \%$ ), progressive faults may go undetected for a long time, thus endangering the entire system. In some cases, progressive faults may turn into failures. It is our contention that AR-modeling of the residuals provides a reliable and sensitive indicator of the fault before the fault becomes apparent in the raw residuals; in this technique, the number of false alarms is greatly reduced.

\section{CONCEPT OF The Main Approach}

According to the literature (Isermann and Balle, 1997; Chen and Patton, 1999), the term "fault" is defined as unpermitted deviation of at least one characteristic property or parameter of the system from acceptable/usual condition, while the 
term "failure" refers to complete breakdown of a system component or function. A fault may lead to a failure and while we cannot prevent many faults, by detecting them as early as possible, we may be able to prevent a failure. A progressive fault (or so-called incipient fault in some fields) is considered in this work. It is a very slowly developing fault, that is, it denotes an abnormal condition that gets worse and worse until some system component totally fails or some physical quantity (speed, temperature, pressure, etc.) exceeds allowable limits. For example, it can be described as follows:

$$
f(t)=\beta(t-T) f_{0}
$$

where $T \geq 0$ is the beginning time of a fault, $f_{0}$ is a constant vector, and $\beta(t-T)$ is defined as follows:

$$
\beta(t-T)= \begin{cases}0 ; & \text { if } \quad t \leq T \\ 1-e^{-k(t-T)} ; & \text { if } \quad t \geq T\end{cases}
$$

with $k \geq 0$ being a small constant. The fault $f(t)$ becomes a failure when it exceeds some threshold $M$ at a specific time $t^{*}:$ if

$$
f\left(t^{*}\right)=M
$$

and

$$
f(t) \geq M \quad \forall t \geq t^{*}
$$

then a failure has occurred at time $t=t^{*}$.

Remark 1: The above fault model is given only for illustration purposes; the technique used in this paper works regardless of any specific fault model.

Because of the nature of a progressive fault, it is much more difficult to detect earlier, compared with an abrupt fault. The goal of this paper is detect such faults as soon as possible even when they are tolerable at the early stage, to prevent any serious consequences. That is, detect the fault before it becomes a failure.

In this work, the model of the closed loop dynamic system including the controller is assumed to be known. (This assumption is used only for simplicity; if the system model is not known, then system identification methods can be used to generate the necessary residuals. Regardless of how the residuals are created, our method can be applied on the residuals directly.) Let the output of the system model be $y_{M}(k)$, and the actual plant output be $y_{k}$, as shown in Figure 1; the nominal (fault-free) plant output is the same as the model output. The residual is given by

$$
R(k)=y(k)-y_{M}(k) .
$$

If the output signal can only be measured with a certain noise $v_{1 k}$, the noisy residual is

$$
R(k)(n o i s y)=y(k)-y_{M}(k)+v_{s}(k) .
$$

Suppose the controller output (the manipulated variable) obtained from the model is $u_{M}(k)$, and the actual controller output is $u(k)$, as shown in Figure 1. Assuming that the

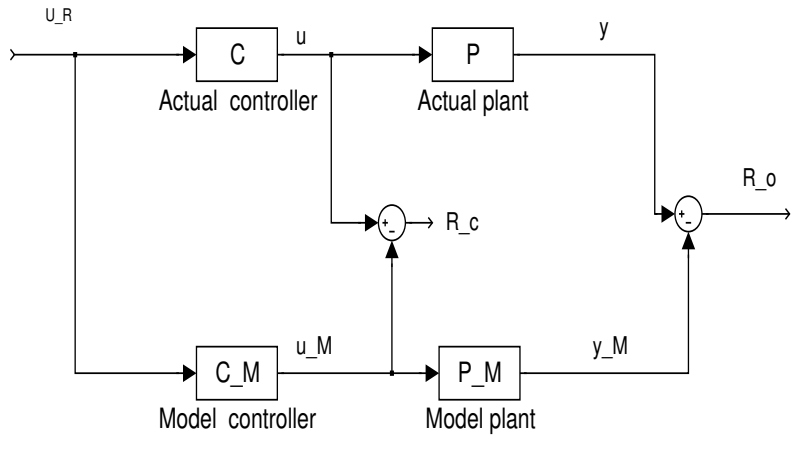

Fig. 1. Block diagram of residual generation

actuator signal can be measured, the "controller residual" is given by

$$
R_{c}(k)=u(k)-u_{M}(k) .
$$

If the controller output can only be measured with a certain noise $v_{c}(k)$, then the noisy controller residual is

$$
R_{c}(k)(n o i s y)=u(k)-u_{M}(k)+v_{c}(k) .
$$

Clearly, during no-fault situations the "true residuals" remain zero. When the measurements are available without any noise, the generated residuals are sufficient to indicate the presence of a fault - either progressive or sudden - in the system. However, when the measured signals contain random measurement noise, the raw residuals do not immediately show the presence of the fault. In such cases, we propose to use the AR modeling technique to extract the "true residuals", and use that variable as the main indicator of system fault. Essentially, the AR modeling provides a way of filtering out the noise from the residuals. It is worth pointing out that front-end filtering can also be used to reduce sensor noise. Although a low-pass filter is easy to implement, its performance is not very good, since some of the low-frequency noise will always remain in the filtered signal. Another way to filter the noise would be to take a moving average of the signal; however, since the zero mean of the signal can only be obtained (theoretically) with an infinite number of data points, and any practical implementation of the moving average must have a finite window size, we cannot expect a true mean value from this method. Using the AR modeling, in theory, the AR parameters should be zero; thus, the AR-processed signal is also zero. In practice, the AR parameters estimated in real time will be small non-zero numbers.

Remark 2: The AR-processed residual is not viewed as a random number. The idea is that this technique essentially replaces the stochastic indicator (raw residuals) by a deterministic one.

\section{AutoRegressive (AR) Model of Processes}

Claim 1: As long as the residuals constitute a zero-mean uncorrelated random process, any modeling of the residuals should yield an output which is very close to zero, since it 
is fundamentally impossible to predict a random sequence. As soon as there is a fault, the residuals will contain the information in some form; at this time, the information can be extracted with a simple AR model.

The AR model of a process $z_{k}$ is given by

$$
z(k)=\sum_{i=1}^{n} \alpha_{i} z(k-i),
$$

where $\alpha_{i}$ are the AR parameters and $n$ is the order of the AR model. In this work, the process $z_{k}$ can be the residuals collected either at the system output or the controller output, depending on which signal is deemed to be more sensitive to the faults. Note that during the no-fault period, assuming the availability of correct models for the plant and the controller, the residuals will be either:

- zero, if the measurements are non-noisy, or

- equal to the measurement noise, if the measurements are noisy.

In the noise-free case, there is no added benefit to using the AR modeling: the raw residuals at the system output and the controller output can serve as fault indicators. If the measurements are noisy, then the raw residuals can mask the presence of faults, at least temporarily; in such cases, the AR-predicted "true residuals" are a much better indicator of faults. In this work, we propose the use of the Kalman filter for the parameter estimation; all the appropriately modified equations for the implementation of the Kalman filter in this specific problem are presented in the next section.

\section{A. AR Parameter Estimation in Real Time}

Let the M-dimensional vector $x(k)$ denote the state of a discrete-time, linear dynamical system and let an $\mathrm{N}$ dimensional $y(k)$ denote the observed signal of the system, both measured at time $\mathrm{k}$. Thus the system model is described by two equations. The process equation is given by

$$
x(k+1)=A(k+1, k) x(k)+v_{1}(k)
$$

where $A(k+1, k)$ is known by $\mathrm{M}$-by -M state transition matrix relating the states of the system at times $\mathrm{k}+1$ and $\mathrm{k}$. $v_{1}(k)$ is modeled as zero mean, white noise process.

The measurement equation of the system is given by

$$
z(k)=C(k) x(k)+v_{2}(k)
$$

where $C(k)$ is a known $\mathrm{N}$-by-M measurement matrix. The noise vectors $v_{1}(k)$ and $v_{2}(k)$ are assumed to be uncorrelated. The state transition matrix $A(k+1, k)$ and the measurement matrix $C(k)$ are also assumed to be known. Given this dynamic system model, a Kalman filter can estimate the state vector $x(k)$ based on noisy measurements $z(k)$. The covariance matrices of the noise signals are assumed to be known; if they are not known, they can also be estimated in real time.
In order to estimate the AR parameters of the residual sequence, let us map the process Eq. 6 and measurement Eq. 7 to the AR model. More specifically, by letting

$$
\begin{gathered}
x(k)=\alpha(k)=\left[\alpha_{1}(k), \cdots, \alpha_{n}(k)\right] ; \\
C(k)=[z(k-1), \cdots, z(k-n)] ;
\end{gathered}
$$

and $A(k+1, k)=I_{n \times n}$, we get the following measurement and update equations respectively.

$$
\begin{aligned}
& \alpha(k+1)=\alpha(k)+v_{1}(k) \\
& z(k)=C(k) \alpha(k)+v_{2}(k)
\end{aligned}
$$

Now, state vector $x(k)$ of the dynamic system contains the AR parameters $\alpha_{i}$, and can be estimated in real time. The update equation for the AR parameters obtained from Eq.9, with the computed Kalman gain at each time step $K(k)$ is:

$$
\hat{\alpha}(k)=\hat{\alpha}(k-1)+K(k)(z(k)-\hat{z}(k)),
$$

where $\hat{z}(k)$ is the estimated output from the previous steps of the estimation process (using the AR parameters estimated at time $k-1)$. The standard Kalman gain $K(k)$ is given by

$$
K(k)=A P C^{\tau}\left(C P C^{\tau}+R\right)^{-1}
$$

where the error covariance matrix

$P(k) \triangleq \mathbf{E}\left[(z(k)-\hat{z}(k))(z(k)-\hat{z}(k))^{\tau}\right]$ is updated by

$P(k+1)=A P(k) A^{\tau}+Q-K(k)\left(C P(k) C^{\tau}+R\right) K^{\tau}(k)$

with $R$ and $Q$ being covariance matrices of the measurement noise and process noise respectively, that is

$$
\mathbf{E}\left(\left[\begin{array}{l}
v_{1}(k) \\
v_{2}(k)
\end{array}\right]\left[v_{1}^{\tau}(j) v_{2}^{\tau}(j)\right]\right)=\left[\begin{array}{cc}
Q & 0 \\
0 & R
\end{array}\right] \delta_{k j}
$$

where $\delta_{k j}$ is the Kronecker delta function, $\mathbf{E}(\cdot)$ stands for the expectation operator, $A$ is an identity matrix in this case. Note that due to the random walk nature of the process equation, Eq. (8), the AR parameters are allowed to change in small amounts, and this allowance is controlled by the covariance of process noise used in the algorithm.

The fault detection process in this approach consists of extracting fault information from the noisy residuals. Indeed, substituting $E\left[v_{2}(k)\right]=0$ in (9) we get

$$
E[z(k)]=C(k) E[\alpha(k)],
$$

which also means that $\hat{z}(k)=C(k) \hat{\alpha}(k)$; this is the quantity we use as the actual fault indicator. Ideally, nonzero $\hat{z}(k)$ indicates occurrence of a fault. In practice, we establish a threshold for the decision rule; the threshold depends on the definition of "failure" in each application, and may also depend on other application-specific quantities. 


\section{B. Closed-loop vs. Open-loop Cases}

For early detection of progressive faults that have the potential to turn into failures, we must select carefully the best location for generating the residuals. It should be noted that a similar issue - that is, choice of the best sensor location - has been investigated in ( $\mathrm{Xu}$ and Jiang, 2000). In that paper, the authors concluded that the controller output is the optimal location to obtain the information about small parameter changes or faults in the actuator, feedback sensor and the plant. Although they did not consider progressive faults, and they considered noisefree cases only, it is our contention that the controller output is the best location for residual generation in all closedloop cases; in addition, the more effective the controller is, the more important it is to base the fault detection on the controller-output residuals and not on the system-output residuals. Our experiments show that the AR-predicted values obtained from the controller-output residuals constitute the best indicator of progressive faults in a closed-loop system. Also, a restrictive assumption about norm bound of the transfer functions in certain frequency ranges is critical in ( $\mathrm{Xu}$ and Jiang, 2000), while it is not required in this paper. We demonstrate that AR-predicted residuals at the controller output constitute the best indicator in a closedloop system. To illustrate our concept, a series of computer simulations have been conducted on an aerospace system example, which is described in the next section.

Remark 3: A straight forward extension of the method to the ARMA (AutoRegressive Moving Average) modeling will result in the estimate of the transfer function between the input to the system and the residual signal. This transfer function would be a direct estimation of the fault in the system, which is useful for fault accommodation as pointed out by (Patton, 1997; Staroswiecki and Gehin, 2001).

In the next section, results of computer simulation studies that demonstrate the application of our concept are presented.

\section{A BOEING 747 AIRCRAFT EXAMPLE}

In this section, a Boeing 747 jet transport aircraft model is used to test the proposed method in this paper. Using the geometrical and aerodynamic data for the Boeing 747 jet transport aircraft from (Etkin and Reid, 1994) and considering flight condition: cruising in horizontal flight at 40,000 feet with Mach number 0.8, the dynamics of longitudinal model is characterized by the following matrix:

$$
\begin{aligned}
& A=\left[\begin{array}{cccc}
-0.006868 & 0.01395 & 0 & -32.2 \\
-0.09055 & -0.3151 & 773.98 & 0 \\
0.0001187 & -0.001026 & -0.4285 & 0 \\
0 & 0 & 1 & 0
\end{array}\right] ; \\
& B=\left[\begin{array}{cc}
-0.000187 & 9.66 \\
-17.85 & 0 \\
-1.158 & 0 \\
0 & 0
\end{array}\right] ; \quad C=\left[\begin{array}{cccc}
1 & 0 & 0 & 0 \\
0 & 1 & 0 & 0 \\
0 & 0 & 1 & 0 \\
0 & 0 & 0 & 1
\end{array}\right] .
\end{aligned}
$$

where the state vector and control/command vector are composed by:

$x_{1}=v-$ the longitudinal velocity;

$x_{2}=\omega-$ the vertical velocity;

$x_{3}=\varpi_{y}-$ the rate of pitch;

$x_{4}=\theta-$ the pitch angle,

$u_{1}=\delta_{e}-$ the elevator command;

$u_{2}=\delta_{a}-$ the throttle command.

First, a fault-free nominal model of the entire system is used to generate nominal outputs etc., which are to be used as the reference for residual generation. It is assumed that a fault occurred at the execution level, for example, at the servomotor of the elevator channel (because of a low supply voltage or mechanical brake). The simulated fault is of a slowly developing nature and occurs at $\mathrm{t}=4 \mathrm{sec}$, which is depicted in Figure 2. We assume that this fault becomes a "failure" at time $t \geq 10 \mathrm{sec}$, therefore our goal is to see whether we can detect the fault at or before this time.

The values of the residuals at two points in the system are measured: (a) the system output, that is, the longitudinal velocity, and (b) the controller output, that is, the elevator command. The true residuals under noise-free environment are shown in Figure 3 and Figure 4 respectively. It can be seen that the controller output residual would detect the fault very quickly. Figures 5-6 show the two residuals when the measured signals contain white noise. It is evident that in the noisy case, the raw residuals do not clearly indicate the presence of the fault in the beginning of the process. If the threshold is set to be small, there are too many false alarms. If we set a high detection threshold (see Figure 5) to reduce the number of false alarms, the fault detection time will be delayed till $\mathrm{t}=11 \mathrm{sec}$.

Next, we perform a second-order AR modeling of the residuals at controller output and system output for the noisy case. The results of AR modeling is shown in Figures 7-8 respectively. Figure 7 shows that, by using residual autogression at the system output, the detection threshold can be made small without increasing the rate of false alarms. Even better results can be seen in Figure 8 by using residual autogression at the controller output, where the threshold can be nearly zero (near-perfect noise filtering) and earlier fault detection can be achieved.

The first order equation governing the AR modeling of the residuals at the system output for the noise free case is given by

$$
z(k)=a_{1} z(k-1) .
$$

After the coefficient is estimated $\left(\hat{a}_{1}\right)$ by an ordinary Kalman filter, the AR-predicted residual is given by:

$$
\hat{z}(k+1)=\hat{a}_{1} z(k) .
$$

This process is repeated at each time-step $k$, where the covariance matrices for process, sensor noise sequences and initial estimation error are :

$$
Q=0, \quad R=5 I_{n}, \quad P(0)=0.05 I_{n} .
$$


TABLE I

FALSE ALARM RATES USING RAW RESIDUALS AND AR-PREDICTED ONES AT SYSTEM OUTPUT: DETECTION TIME $=10 \mathrm{SEC}$.

\begin{tabular}{c|c|c}
\hline slopes of fault & raw residual & AR-predicted residual \\
\hline 0.01 & $32.5 \%$ & $7.5 \%$ \\
\hline 0.02 & $15 \%$ & $2.5 \%$ \\
\hline 0.03 & $2.5 \%$ & $0 \%$ \\
\hline
\end{tabular}

TABLE II

FALSE ALARM RATES USING RAW RESIDUALS AND AR-PREDICTED ONES AT CONTROLLER OUTPUT: DETECTION TIME $=7$ SEC.

\begin{tabular}{c|c|c}
\hline slopes of fault & raw residual & AR-predicted residual \\
\hline 0.01 & $22.5 \%$ & $2.5 \%$ \\
\hline 0.02 & $12.5 \%$ & $0 \%$ \\
\hline 0.03 & $2.5 \%$ & $0 \%$ \\
\hline
\end{tabular}

Another similar Kalman filter is utilized at the controller output node, producing AR-predicted residuals, where $P(0)$ is chosen as $0.1 I_{n}$. From the plots of these AR-predicted residuals, we can determine that the slowly developing fault is detectable more clearly from the AR-predicted residuals than from the raw residuals. Moreover, it can also be seen that the controller output residuals are a better indicator of the fault than the output residuals.

In order to investigate the relative frequency of false alarms in the raw residuals vs. the AR-predicted residuals, we have done simulations for incipient faults that occurred at $\mathrm{t}=4$ sec, with different slopes. Comparison of false alarm rates between raw residuals and their AR modeling at system output is summarized in Table 1 where the detection time is fixed at $\mathrm{t}=10 \mathrm{sec}$, while Table 2 compares false alarm rates between raw residuals at controller output and the ARpredicted ones where the detection time is fixed at $\mathrm{t}=7 \mathrm{sec}$. From the above tables, it can be seen that the AR-predicted residuals significantly reduce the false alarm rates, regardless of which residuals are being used. Moreover, comparing table 1 and table 2, it is clear that the AR-predicted residuals at the controller output detect the faults earlier, with very little false alarms.

\section{CONClusion}

In this paper, we have presented a simple and practical way of creating a fault indicator that is very useful for early detection of progressive faults. This fault indicator is the AR-predicted value of the residual signal. We have demonstrated that in closed-loop systems, autoregression of the residuals at the controller output provides the best and earliest indicator of the presence of a fault. In future work, we plan to implement methods of estimating the transfer function between the residual signal and the system input; this transfer function should provide an estimate of the fault, which would be useful for fault accommodation and potentially, for design of active fault-tolerant control.

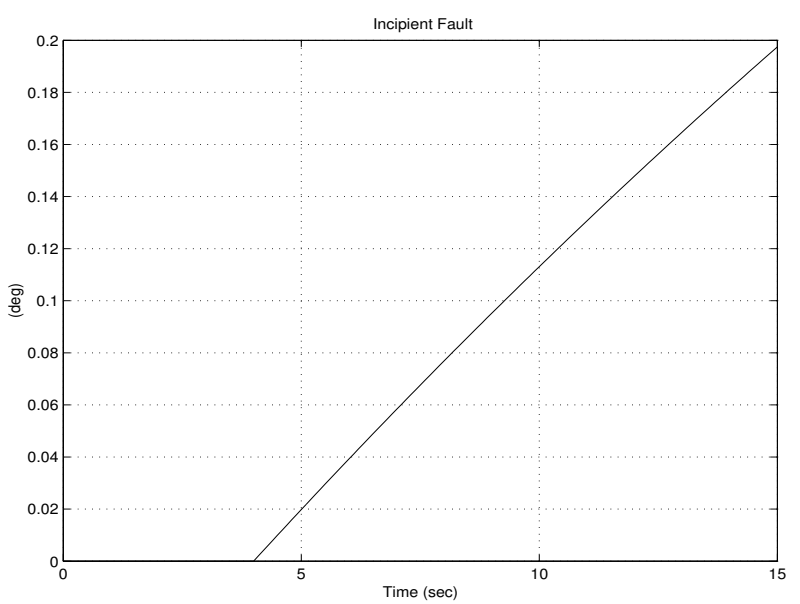

Fig. 2. The incipient fault signal $f(t)$

\section{ACKNOWLEDGMENTS}

This work is partially supported by NASA under award NCC5-573, contract NASA/LEQSF(2001-04)-1. The authors thankfully acknowledge the initial simulation studies performed by Mr. Sundara Kumar, a graduate student at the Center for Advanced Computer Studies at UL Lafayette.

\section{REFERENCES}

[1] Gertler, J.J., "Survey of model-based failure detection and isolation in complex plants", IEEE Control Systems Magazine, Vol. 8, 1988, pp. 3-11.

[2] Frank, P.M. and X. Ding and B. Koppen-Seliger, "Current developments in the theory of FDI", Proc. IFAC Safeprocess, Budapest, Hungary, 2000, pp. 16-27.

[3] Basseville, M. and I. Nikiforov, Detection of abrupt changes - Theory and Applications, Prentice Hall Information and System Sciences Series, Englewood Clifs, N.J., 1993.

[4] Chen, J. \& R. J. Patton, Robust model-based fault diagnosis for dynamic systems, Kluwer Academic Publishers, 1999.

[5] Silvio, S., C. Fantuzzi and R.J. Patton, Model-based Fault Diagnosis in Dynamic Systems Using Identification Techniques, SpringerVerlag, UK, 2002.

[6] Demetriou, M. and M.M. Polycarpou, "Incipient fault diagnosis of dynamical systems using online approximators", IEEE Transactions on Automatic Control, Vol. 43, 1999, pp. 1612-1617.

[7] Parlos, A.G., K. Kim and R. Bharadwaj, "Detection of induction motor faults - combining signal-based and model-based techniques", Proc. of American Control Conference, Anchorage, 2002, pp. 45314536.

[8] Keller, J.Y., "Fault isolation filter design for linear stochastic systems", Automatica, Vol. 35, 1999, pp. 1701-1706.

[9] Chowdhury, F.N., "Ordinary and neural chi-squared tests for fault detection in stochastic Systems", IEEE Transactions on Control Systems Technology, Vol. 8, 2000, pp. 372-379.

[10] Isermann, R. and P. Balle, "Trends in the application of modelbased fault detection and diagnosis of technical processes", Control Engineering Practice, Vol. 5, 1997, pp. 709-719.

[11] Etkin, B. and L.D. Reid, Dynamics of Flight, Stability and Control, John Wiley \& Sons, New York, 1994.

[12] Patton, R.J., "Fault tolerant control: The 1997 situation", Proc. IFAC Safeprocess, Hull, UK, 1997, pp. 1033-1055.

[13] Staroswiecki, M. and A.-L. Gehin, "From control to supervision", IFAC Annual Reviews in Control, Vol. 25, 2001, pp. 1-11.

[14] Xu, Y.H. and J. Jiang, "Optimal sensor location in closed-loop control systems for fault detection and isolation", Proc. of American Control Conference, Chicago, 2000, pp. 1195-1199. 


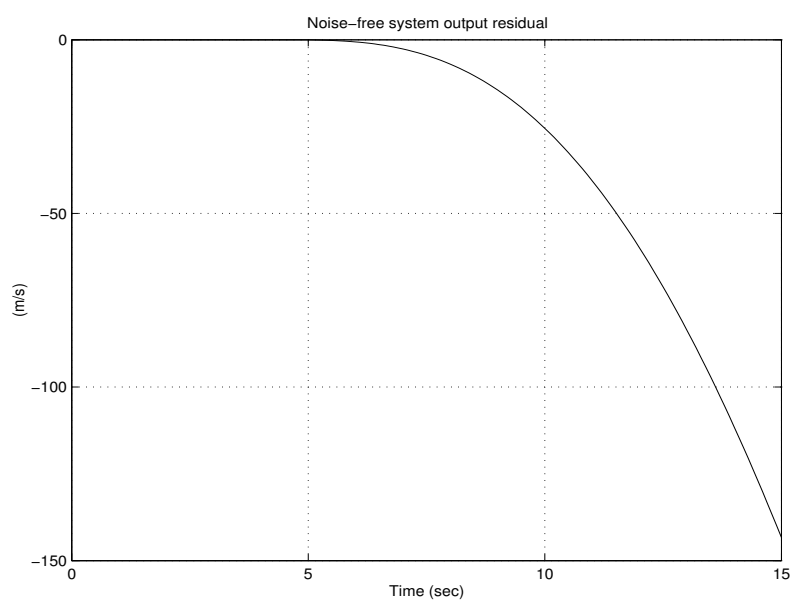

Fig. 3. System output residual: noise-free case

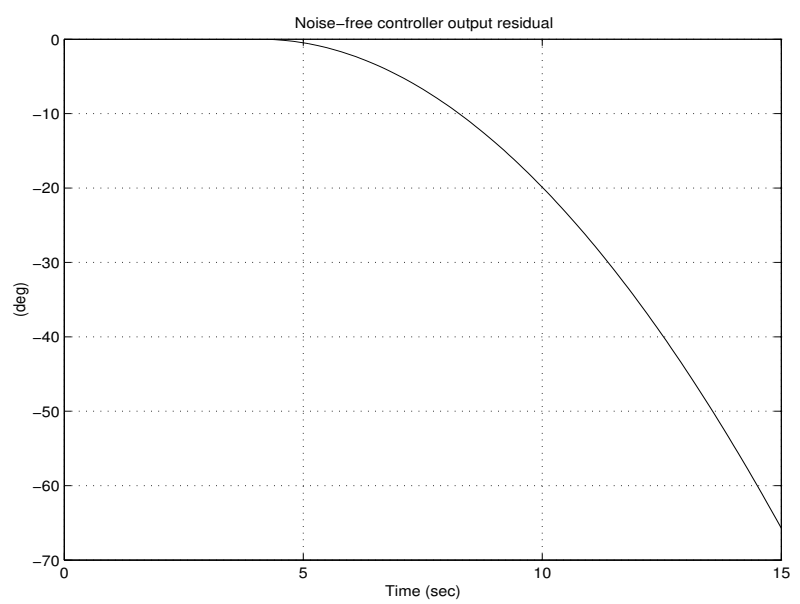

Fig. 4. Controller output residual: noise-free case

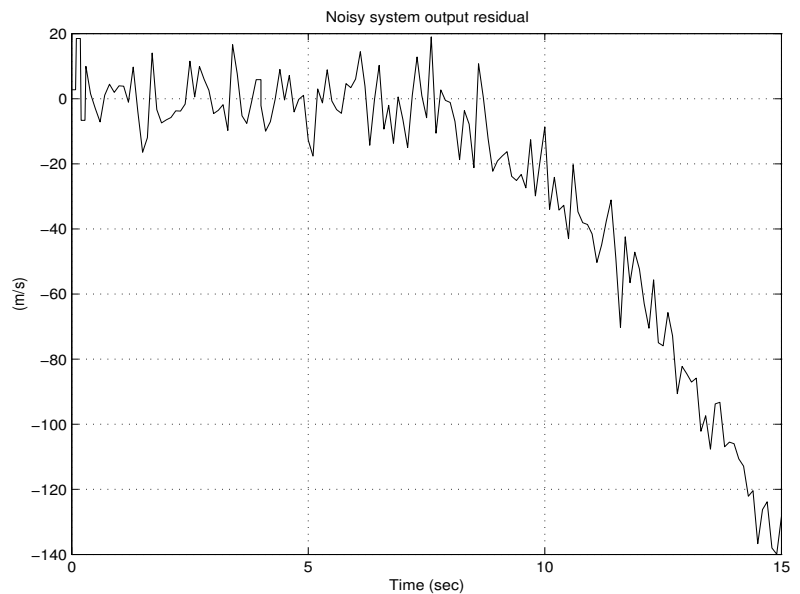

Fig. 5. System output residual: noisy case

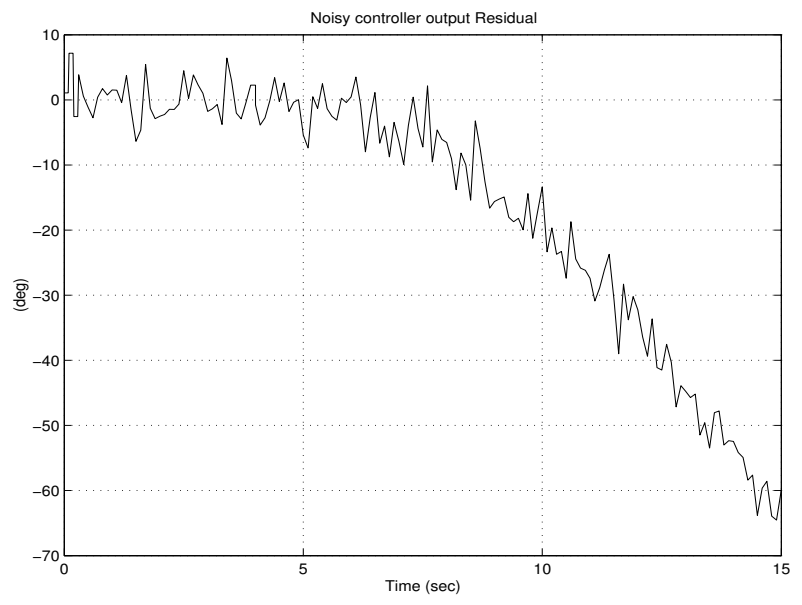

Fig. 6. Controller output residual: noisy case

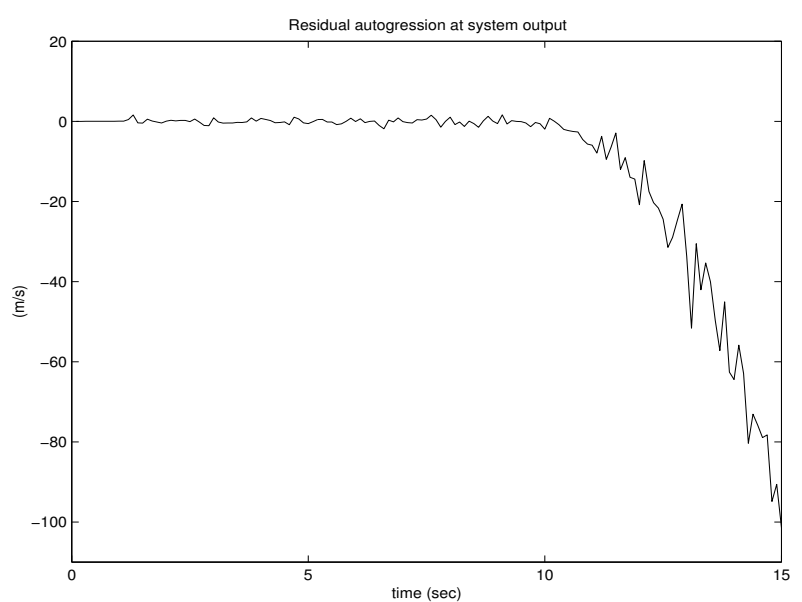

Fig. 7. AR-predicted system output residual

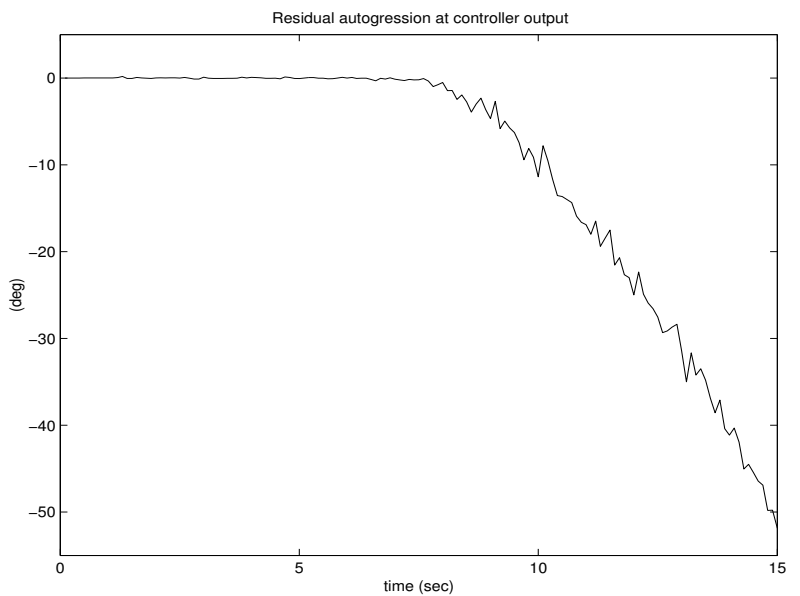

Fig. 8. AR-predicted controller output residual 